\title{
TNT: Touch 'n' Tangibles on LC-Displays
}

\author{
Ramon Hofer and Andreas Kunz \\ ETH Zurich, Institute for Machine Tools and Manufacturing \\ Tannenstrasse 3, 8092 Zurich, Switzerland \\ \{hofer, kunz\}@inspire.ethz.ch
}

\begin{abstract}
In this paper, we present TNT (Touch 'n' Tangibles) - a new combination of several existing hardware technologies, which are integrated into an LC-display. TNT enables users to interact using finger touch and tangible user interfaces at the same time on an active flat panel screen, while maintaining precise identification of all interactive objects and fingers. TNT can accurately distinguish between touch and Tangible User Interfaces input by assigning different time slots to each interactive object using the same sensing technology for both methods. TNT's tracking is not affected by objects on the screen other than fingers and active Tangibles User Interfaces, which makes it ideal for use in brainstorming applications.
\end{abstract}

Keywords: HCI, Tracking, Sensor, Input Device, Multi Touch, TUI, Interaction.

\section{Introduction}

There already exists a wide range of multitouch detecting hardware on the market, which can be applied to small (up to 22") LC-displays. Looking into the field of research prototypes, even more technologies are available. Research in detecting multitouch interaction reaches back to the mid 1980s, when Lee et al. [1] already designed a multi-touch tablet surface with front projection. Up to now, many new technologies were developed. One of these is DiamondTouch [2], which uses capacitive sensing of the user's fingers. SmartSkin [3] uses a similar antenna grid that can detect signal distortion on the grid by close objects. HD Touch [4] can detect reflections of touches through an LC-matrix by using a webcam and an infrared illumination. Since 2005, the FTIR (Frustrated Total Internal Reflection) multitouch detection principle [5] has inspired a lot of researchers to improve this back-projection technology or to adapt it to larger installations or other display devices such as Liquid Crystal Displays (LCD) [6].

Systems which support both - touch and TUI (Tangible User Interface) detection with reliable object identification - are not very common. Such an integrated system is Slap [7], which uses the FTIR principle on a back-projection table. It is capable of detecting touch and TUIs with attached silicone pads. Since silicone can be used to couple light out of an FTIR screen [8], it can be used as TUI identifier pads. Other projection-based technologies such as PlayAnywhere [9] use webcams above the screen to track objects. ReacTable [10] is one of the first and widely spread technologies that can simultaneously detect touch and TUI positions and orientations. Infrared 
light from IR (Infrared) emitters behind the interactive screen is reflected by the devices' fiducials on the screen. This allows unambiguously identifying each device. Touch can be detected as well by an interpretation of reflected blobs caused by the fingertips.

In the near future, interactive systems will most likely be based on LC-display technology. Flat screens allow applications on tables, at walls, and anywhere where the size is crucial. Thus, research increasingly focuses on interactive systems that support various methods of interactive input. ThinSight [11] is one of the first technologies that can detect touch and TUI interaction on an LC-display. The principle is similar to that of the ReacTable [10], but the screen is an LC-matrix and the camera was replaced by IR-sensors mounted in a discrete array directly behind the LC-matrix. An IR emitter is mounted next to each sensor. The emitted IR light is reflected by objects in front of the screen. Tracking update rates are around $10 \mathrm{~Hz}$.

Our 'Touch and Tangibles' system (TNT) is also integrated into an LC-display. It can detect passive touch points and active TUI information (position, orientation, status) at high speed $(150 \mathrm{~Hz})$ by using a time-multiplexing. Additionally, passive objects other than fingers on the surface are not detected by TNT. This allows placing everyday's objects like coffee mugs, laptops, books, etc. on the screen without disturbing the tracking. We consider this a main advantage over existing systems, since we aim to support natural and intuitive group work. Applications such as collaborative sketching, brainstorming, etc, in which a high level of creativity and spontaneity is required, should not depend on the user's ability to find out the systems behaviour.

\section{System Description}

TNT basically consists of two technologies that are integrated into an LC-display (see Figure 1). For touch detection, an FTIR overlay is used, which acts as an IR light source. The second component is the detection array, which consists of IR sensors being mounted in a two-dimensional array behind the LC-matrix and the FTIR overlay. The detection array receives signals either from decoupled IR light of the FTIR overlay, or from synchronized active TUIs also emitting IR light.

\subsection{Touch and Device Detection}

An acrylic panel is mounted in front of the LC-matrix, which displays the image on the screen. IR LEDs mounted at the side of this panel couple light into the acrylic and due to the properties of refraction of acrylic and air most of the IR rays are totally internally reflected.. As soon as a finger or another "soft" coupling object is placed on the surface of the acrylic, light rays are scattered out of the acrylic and exit the overlay towards the underlying sensor array as presented in [6]. Special considerations were made concerning the sensor-sensor distance, since this significantly influences the detection performance. Here, a sensor distance of $10 \mathrm{~mm}$ in an overlay's distance of $15 \mathrm{~mm}$ was chosen. This ensures that rays decoupled by finger touches trigger sufficient sensors to perform an interpolation between the analog sensor values for the determination of an exact touch position. 


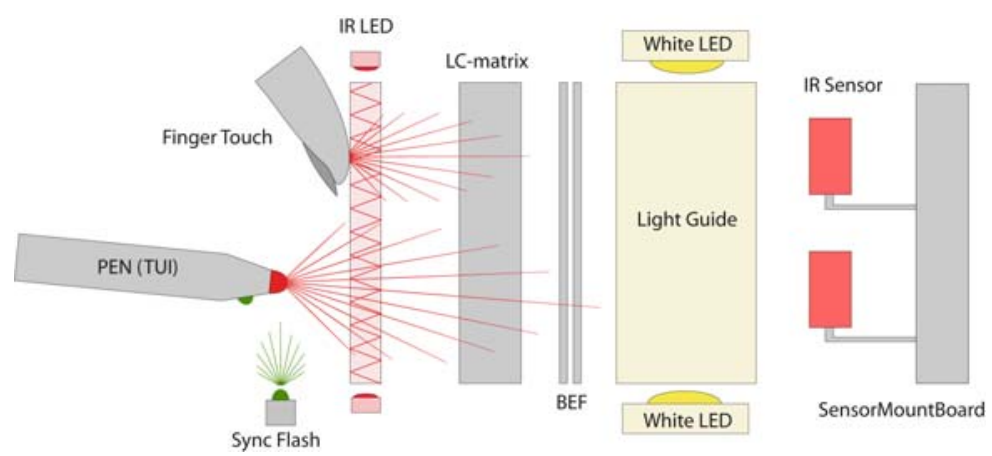

Fig. 1. Components of TNT

The device detection on TNT is basically trivial. An active device (IR light source) radiates through all the components until it hits upon the IR sensors. This cascade of components absorbs a relatively large amount of the incident IR-light. Since the radiation cone of the device's LED triggers multiple sensors at a time, the position of the device can be computed by interpolation using a predefined (measured) interpolation curve.

Two methods to distinguish between several active devices have been considered: Time- or space-multiplexed separation. If space multiplexing is used, the algorithm has to separate the sensor values into groups of potential devices. This can only be done, if the devices are not too close to each other, since otherwise (if the cones are almost overlapping) no exact assignment can be computed. This special situation can occur, if two pens are hovering and pointing at the same location. Additionally, for the space multiplexed method, the device might not be identified uniquely if removing and entering the active area does not occur at the same location. Thus, misinterpretations can easily occur.

By using the time multiplexed separation, these effects can be avoided. Each synchronized device is assigned to a separate timeslot, in which only one device is active. If the tracking is fast enough, the user will not notice the time delay for each device's tracking update. The amount of devices can easily be extended. Within TNT, a time multiplexed separation is used. A synchronization flash ensures that all the devices run in sync at any time. A slightly longer synchronization signal is used as the start frame in order to keep the logic of all devices consistent. In order to combine touch and TUI interaction using the same sensors, a timeslot for touch detection is additionally inserted into this synchronized sequence after the last TUI's timeslot. During this frame, no active device is triggered and only the FTIR-LEDs are turned on. Sensors will only receive an input from passive touch devices (fingers) on the surface in this particular timeslot.

The proposed time multiplexed detection method also permits an insertion of additional frames per TUI to transmit information such as the state or other additional data. For example, a device can be equipped with a switch that can be pressed by the user. This would alter its state to "pressed". Thus, it is possible to distinguish between a hovering (tracking) state and a pressed (dragging) state. When the TUI is removed from the screen, it remains in an "out of range" state according to [12]. 


\section{Proof of Concept Setup and Performance}

TNT is integrated into a modified 15" LC-display and uses modular sensing hardware. A SensorModule consists of two SensorControlBoards (SCB) and one SensorMountBoard (SMB). On one SMB, 128 Sensors are aligned in a $10 \mathrm{~mm}$ grid, resulting in an active detection area of $160 \times 80 \mathrm{~mm}$. In total, two of these modules are mounted behind the diffuser of an LC-display, realizing a total detection area of $160 \mathrm{x}$ $160 \mathrm{~mm}$ (see Figure 2). An SPI Bus connects all SCB to the MasterControlBoard (MCB). The MCB handles all the synchronization logic, USB data preparation, and data retrieval from all the SCB. Whereas a SCB only converts the analog sensor voltage levels into digital values and filters relevant data out of the sensor values.
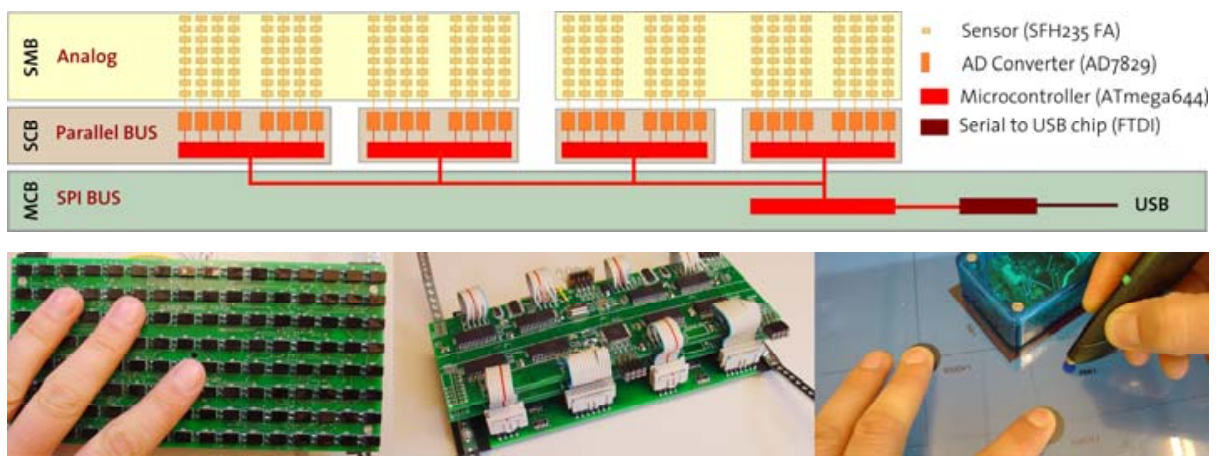

Fig. 2. Top: Schematic of two Modules $(1$ Module $=1$ SensorMountBoard $(\mathrm{SMB})+2$ SensorControlBoardd (SCB); Bottom: Technical proof of concept and user interaction

The FTIR touch overlay is a $3 \mathrm{~mm}$ thick acrylic. At every flange of the acrylic, IR SMD LEDs ( $880 \mathrm{~nm}$ wavelength) are mounted (distance of $7 \mathrm{~mm}$ from LED to LED). The interaction devices have an embedded microcontroller that receives the modulated synchronization pulses via an infrared sensor. The microcontroller counts the pulses and sends the corresponding bit codes via one or two (if orientation is needed) IR LEDs to the TNT system.

For the proof of concept, we have set up a small visualization software which shows graphical representations of all used TUIs together with their positions, states, and orientations. The software reads all available sensor values and computes the corresponding interpolated centers of the TUIs. For the multitouch frame, a blob detection algorithm is performed on the sensor values, which separates the touch points in different groups in order to distinguish between multiple touch points.

At the moment, the system is set to track 6 different devices (each using 2 frames) and multitouch input (one frame). This results in 13 timeslots (frames).

For the acquisition and transfer of all sensor values within one frame, approximately $0.5 \mathrm{~ms}$ are needed (depending on the amount of active sensors). This results in a system inherent frame rate of around $2 \mathrm{kHz}$. Depending on the amount of used frames, the effective update rate for the detection of all devices including multitouch drops to around $150 \mathrm{~Hz}$. 
The accuracy of touch and LED center detection is around $1 \mathrm{~mm}$. The detection is very robust. Since time multiplexed detection is used, no misinterpretations occur. Touch and TUI detection can even be used concurrently. For example: Two pens in the hovering state could point at the same position and still do not affect each other's position detection.

Since an overlay is used for the multitouch detection, the offset between interaction and display layer is $3 \mathrm{~mm}$, which is not distracting, but noticeable.

Image quality of the system is not as good as on the original LC-display due to the acrylic overlay and removal of two BEF (brightness enhancement films), Thus, the sensors are slightly visible behind the image display.

As with all IR-based systems, direct sunlight is another drawback of TNT and makes it impossible to reliably track any TUI or touch.

\section{Conclusion and Outlook}

TNT is a proof of concept that shows that touch and TUI interaction is concurrently possible on LC-displays. The combination of two known technologies shows a new way of technically combining two different interaction metaphors. It supports tracking of position, orientation and state of TUIs, while also being able to track multiple finger touches. By using the widely known FTIR method, the system is immune against detection of most objects other than finger touches.

The proof of concept is still very small $(160 \mathrm{x} 160 \mathrm{~mm})$. Theoretically, it could be scaled up to a 50 inches image diagonal. Then, the IR LED for the FTIR would have to be much more powerful in order to generate enough total internal reflections over the whole interactive area. On the other hand, the hardware is designed in such a way that it can be scaled up to any size although overall complexity would increase potentially and industrial manufacturing of all the PCBs would be necessary.

In the future, it is planned to add more modules to the prototype to increase the active area of tracking. Another important aspect of improvement is image quality. Different films have to be tested to find a good solution for reflecting a lot of visible light in front of the sensors but still let pass through infrared light. It is also planned to mount the synchronization flash inside the screen (behind the LC- matrix) to make the TNT even more robust and flexible. However, the tracking and detecting algorithm already works very well under normal office lighting conditions.

\section{Acknowledgements}

We want to thank the "Institute of Machine Tools and Manufacturing" for the support and Marlis Etter for helping with soldering the electronic boards.

\section{References}

1. Lee, S., Buxton, W., Smith, K.C.: A Multi-Touch three Dimensional Touch-Sensitive Tablet. In: Proceedings of the SIGCHI conference on Human factors in computing systems, vol. 15(5), pp. 21-25. ACM Press, New York (1985) 
2. Dietz, P., Leigh, D.: DiamondTouch: A Multi-User Touch Technology. In: Proceedings of the 14th annual ACM symposium on User interface software and technology, Orlando, Florida, pp. 219-226. ACM Press, New York (2001)

3. Rekimoto, J.: SmartSkin: An Infrastructure for Freehand Manipulation on Interactive Surfaces. In: Proceedings of the SIGCHI conference on Human factors in computing systems, Minneapolis, Minnesota, USA, pp. 113-120. ACM Press, New York (2002)

4. Motamedi, N.: Multi-touch and Object Sensing on a High Definition LCD TV. In: CHI 2008 extended abstracts on Human factors in computing systems, Florence, Italy. ACM Press, New York (2008)

5. Han, J.: Low-Cost Multi-Touch Sensing through Frustrated Total Internal Reflection. In: Proceedings of the 18th annual ACM symposium on User interface software and technology, Seattle, WA, USA, pp. 115-118. ACM Press, New York (2005)

6. Hofer, R., Naeff, D., Kunz, A.: FLATIR: FTIR Multi-touch Detection on a Discrete Distributed Sensor Array. In: Proceedings of the 3rd international conference on Tangible and Embedded Interaction (TEI), Cambridge, UK, pp. 317-322 (2009)

7. Weiss, M., Wagner, J., Jansen, Y., Jennings, R., Khoshabeh, R., Hollan, J.D., Borchers, J.: SLAP Widgets: Bridging the Gap Between Virtual and Physical Controls on Tabletops. In: Proceedings of the 27th international Conference on Human Factors in Computing Systems, CHI 2009, Boston, MA, USA, April 4-9, pp. 481-490. ACM, New York (2009)

8. Smith, J.D., Graham, T.C.N., Holman, D., Borchers, J.: Low-Cost Malleable Surfaces with Multi-Touch Pressure Sensitivity. In: TABLETOP 2007. Second Annual IEEE International Workshop on Horizontal Interactive Human-Computer System, Rhode Island, USA, October 10-12, pp. 205-208 (2007)

9. Wilson, A.D.: PlayAnywhere: A Compact Interactive Tabletop Projection-vision System. In: Proceedings of the 18th annual ACM symposium on User interface software and technology, Seattle, WA, USA, pp. 83-92. ACM Press, New York (2005)

10. Kaltenbrunner, M., Bencina, R.: reacTIVision: A Computer-Vision Framework for TableBased Tangible Interaction. In: Proceedings of the 1st international Conference on Tangible and Embedded interaction, TEI 2007, Baton Rouge, Louisiana, February 15-17, pp. 69-74. ACM, New York (2007)

11. Izadi, S., Hodges, S., Butler, A., Rrustemi, A., Buxton, B.: ThinSight: Integrated Optical Multi-touch Sensing through Thin Form-factor Displays. In: Proceedings of the 2007 Workshop on Emerging Displays Technologies: Images and Beyond: the Future of Displays and Interaction, EDT 2007, San Diego, California, August 4, vol. 252, p. 6. ACM, New York (2007)

12. Buxton, W.: A three state model of graphical input. In: Diaper, D., et al. (eds.) HumanComputer Interaction - INTERACT 2009, pp. 449-456. Elsevier Science Publishers B.V. (North-Holland), Amsterdam (1990) 